\title{
DENSIDADE, AGREGAÇÃO E FRAÇÕES DE CARBONO DE UM ARGISSOLO SOB PASTAGEM NATURAL SUBMETIDA A NÍVEIS DE OFERTAS DE FORRAGEM POR LONGO TEMPO(1)
}

\author{
Osmar Conte ${ }^{(2)}$, Cristiane de Lima Wesp ${ }^{(3)}$, Ibanor Anghinoni ${ }^{(4)}$, Paulo César \\ de Faccio Carvalho ${ }^{(5)}$, Renato Levien ${ }^{(4)} \&$ Carlos Nabinger ${ }^{(5)}$
}

\begin{abstract}
RESUMO
O ajuste da quantidade de animais à oferta de forragem é fundamental para a sustentabilidade de pastagens naturais. $\mathrm{O}$ manejo da oferta de forragem, além de interferir nas espécies vegetais que compõem a pastagem, também pode se refletir nos atributos do solo, como densidade, agregação e conteúdo de carbono. Neste estudo, avaliaram-se a densidade do solo, o diâmetro médio ponderado de agregados e frações de carbono sob pastagem natural, manejada por 22 anos com diferentes intensidades de pastejo, representadas por níveis de oferta de forragem de 4, 8, 12 e $16 \mathrm{~kg}$ de matéria seca para cada $100 \mathrm{~kg}$ de peso de animal vivo. Duas áreas excluídas de pastejo foram incluídas como testemunhas. Verificaram-se alterações nos atributos do solo, estando elas relacionadas às intensidades de pastejo. Essas alterações ocorreram, principalmente, na superfície $(0-10 \mathrm{~cm})$, observando-se aumento da densidade do solo com a redução na oferta de forragem. $O$ diâmetro médio ponderado de agregados do solo e a massa de raízes na camada de 0 a $10 \mathrm{~cm}$ apresentaram comportamento inversamente proporcional ao da oferta de forragem. A labilidade do carbono decresce nos tratamentos conforme diminui a oferta de forragem. $\mathrm{O}$ índice de estoque de $\mathrm{C}$ indica que na oferta de $12 \%$ aumentase o estoque de $\mathrm{C}$ no solo em relação à testemunha, enquanto as demais ofertas diminuem. O índice de manejo de carbono demonstra que as maiores ofertas de forragem (12 e $16 \%$ ) proporcionam melhor qualidade ao sistema em pastagem
\end{abstract}

(1) Recebido para publicação março de 2010 em e aprovado em janeiro de 2011.

(2) Doutorando do Programa de Pós-graduação em Ciência do Solo, Faculdade de Agronomia, Universidade Federal do Rio Grande do Sul - UFRGS. Av. Bento Gonçalves 7712, CEP 91540-000 Porto Alegre (RS). E-mail: agriconte@gmail.com

(3) Mestranda do Programa de Pós-graduação em Zootecnia, Faculdade de Agronomia, UFRGS. E-mail: cristianewesp@yahoo.com.br

(4) Professores do Departamento de Solos, Faculdade de Agronomia, Programa de Pós-graduação em Ciência do Solo, UFRGS. E-mails: ibangui@ufrgs.br; renatole@ufrgs.br

(5) Professores do Departamento de Plantas Forrageiras e Agrometeorologia, Faculdade de Agronomia, Programa de Pósgraduação em Zootecnia, UFRGS. E-mails: paulocfc@ufrgs.br; nabinger@ufrgs.br 
natural, ao passo que na menor oferta (4\%) o sistema está perdendo qualidade e comprometendo a sustentabilidade - demonstrado pela redução substancial desse índice.

Termos de indexação: estoque de carbono, pastejo, campo nativo, atributos físicos de solo.

\title{
SUMMARY: SOIL DENSITY, AGGREGATION AND CARBON FRACTIONS OF AN ALFISOL UNDER NATURAL PASTURE AND DIFFERENT HERBAGE ALLOWANCE
}

\begin{abstract}
The adjustment of animal quantity according to the herbage allowance is fundamental to the sustainability of natural pastures. The management of herbage allowance, besides affecting the plant species that compose the pasture, can also compromise soil properties, such as density and aggregation. This study aimed to evaluate the soil bulk density, weighted mean diameter of soil aggregates and carbon fractions under natural pasture, under varying grazing intensities for 22 years, represented by herbage allowance levels of 4, 8, 12, and $16 \mathrm{~kg}$ dry matter per $100 \mathrm{~kg}$ animal weight. Two ungrazed areas were included as references. Changes in soil properties were found, related to the grazing intensity. These changes occurred mainly in the soil surface layer $(0-10 \mathrm{~cm})$, where soil density increased with decreasing herbage allowance. The behavior of the weighted mean diameter of soil aggregates and the root mass in the layer 0 - $10 \mathrm{~cm}$ were inversely proportional to the herbage allowance. The carbon storage index indicated that under forage supplies of $12 \%$ the soil $C$ stock increased compared to the control, but decreased at other allowance rates. The index of carbon management shows that the highest herbage allowance (12 and $16 \%$ ) improved the system quality of natural pasture, while at the lowest allowance rate (4\%) the system lost quality and sustainability, as demonstrated by the substantial reduction of this index.
\end{abstract}

Index terms: carbon stock, grazing, native grassland, soil physical properties.

\section{INTRODUÇÃO}

A pastagem nativa ainda é o principal substrato forrageiro do sul do Brasil, Uruguai e nordeste da Argentina. Na região Sul do Brasil, particularmente no Estado do Rio Grande do Sul, a pecuária de corte está associada histórica e economicamente à exploração das pastagens naturais desde a introdução de bovinos no início do século XVII. Esse ecossistema pastoril é constituído por espécies nativas, cuja diversidade é caracterizada pela existência de cerca de 400 gramíneas e 150 leguminosas forrageiras (Boldrini, 1997).

A incoerência na administração da disponibilidade de pasto e demanda animal está no centro das causas da falta de persistência das pastagens naturais (Gonçalves, 2007). O manejo incorreto das pastagens naturais, ao longo de muitos anos, resulta em degradação e em acelerado processo de erosão dos solos (Carvalho, 2009). A excessiva utilização da pastagem, por meio de intensidades de pastejo demasiadas, tem causado perda de cobertura vegetal, invasão de espécies indesejáveis, erosão do solo e impacto ambiental negativo (Gonçalves, 2007). O adequado ajuste da carga animal à oferta de forragem é a forma mais eficiente de manter o ambiente com produtividade satisfatória (Carvalho, 2009).

O processo de compactação eleva a densidade e reduz a macroporosidade do solo. Nesse contexto, ocorre o aumento da resistência do solo ao crescimento radicular, principalmente em condições de baixa umidade, e a redução da oxigenação do solo, quando este se apresenta em condições de elevada umidade (Marschner, 1995). Segundo Rosolem et al. (1994), em solo compactado, o sistema radicular não se aprofunda, concentrando-se próximo à superfície. Nessas condições, a planta torna-se mais suscetível a déficits hídricos, com limitada capacidade de absorção de nutrientes em camadas subsuperficiais.

Trabalhos nos quais se avaliam os impactos do manejo de pastagens sobre os atributos físicos do solo demonstram que vários fatores contribuem para variações na porosidade e na densidade do solo. Dentre esses, destacam-se a taxa de lotação utilizada, o tempo de pastejo dos animais e a quantidade de biomassa vegetal sob o solo durante o ciclo de pastejo, bem como o teor de umidade e textura do solo (Trein et al., 1991; Salton et al., 2001). Muller et al. (2001), ao estudarem pastagens cultivadas com diferentes formas de manejo e tempos de formação, observaram que a degradação 
das pastagens está relacionada à menor cobertura do solo. Os resultados obtidos por esses autores indicam que, quanto menor a cobertura sobre o solo, maior será o aumento da densidade deste em sua camada superficial e menores serão a porosidade total e o grau de floculação de argilas.

Bertol et al. (1998), estudando uma pastagem natural submetida a diferentes níveis de ofertas de forragem, em Argissolo, constataram alterações nos atributos físicos do solo, em função das ofertas de manejo estabelecidas. Essas alterações evidenciaramse principalmente na camada superficial do solo, havendo diminuição da taxa de infiltração de água, da porosidade total e da estabilidade de agregados no solo à medida que a oferta de forragem foi reduzida. Aumentos na densidade, microporosidade, resistência à penetração mecânica, diâmetro médio ponderado de agregados e redução da macroporosidade na camada superficial de um Argissolo ocorreram nas áreas pastejadas de campo nativo, em comparação a áreas não pastejadas, segundo estudos realizados por Salton et al. (2008).

No Rio Grande do Sul, a manutenção de intensidade de pastejo equivalente a ofertas de 8 e $12 \%$, respectivamente, para a primavera e restante do ano, resulta em maior produção animal em pastagem natural (Soares et al., 2005). Contudo, quando se prioriza o manejo sustentável das pastagens naturais, a definição do nível adequado de oferta de forragem deve considerar, além dos resultados de produtividade vegetal e animal, as alterações em atributos do solo que possam comprometer sua sustentabilidade, já que variações na estrutura do solo atuam diretamente no desenvolvimento do sistema radicular das plantas e, consequentemente, na sua produtividade, fluxo e armazenamento de água no solo e no ambiente.

Nesse sentido, a fração orgânica do solo composta pelo carbono do solo tem papel fundamental na sua sustentabilidade e dos sistemas produtivos (Boddey et al., 2010). A matéria orgânica do solo pode ser avaliada quantitativamente pelo seu estoque total ou nas diferentes frações em que se apresenta. A caracterização da matéria orgânica por fracionamento físico é menos agressiva à sua estrutura do que os métodos químicos e, por isso, constitui uma ferramenta importante nos estudos de mudanças de uso do solo (Christensen, 1992). Essa técnica tem sido aplicada em estudos de quantificação e caracterização qualitativa dos compartimentos da matéria orgânica (Cambardella \& Elliott, 1992), sobretudo para avaliação do efeito de sistemas de manejo do solo, por meio de índices de estoque de carbono e índice de manejo de carbono (Dieckow et al., 2005). Nessa condição, principalmente o índice de manejo de carbono (IMC) permite verificar, pela alteração da labilidade do carbono, se o sistema está tendendo à sustentabilidade ou à degradação (Souza et al., 2009). A proteção física atua nos estoques de matéria orgânica particulada, a qual tem baixa recalcitrância e pouca interação com minerais do solo (Bayer et al., 2004), sendo essa proteção relacionada aos processos de formação e estabilização de agregados (Pulleman et al., 2005).

Neste estudo, realizado em uma pastagem natural manejada por 22 anos sob distintos níveis de oferta de forragem, investigou-se a hipótese de que diferentes intensidades de pastejo podem causar alterações nos atributos físicos, no estoque e nas formas de carbono do solo, bem como no sistema radicular da pastagem nativa. Para isso, avaliaram-se a densidade, o estado de agregação e o conteúdo total de carbono do solo, sua distribuição no perfil e nas formas particulada e associada a minerais. Também foi avaliada a massa de raízes em camadas, a partir da superfície do solo.

\section{MATERIAL E MÉTODOS}

O estudo foi realizado em área de pastagem natural pertencente à Estação Experimental Agronômica da Universidade Federal do Rio Grande do Sul, na região fisiográfica da Depressão Central do Estado do Rio Grande do Sul (3005' S; 51 40' O). A área experimental compreende aproximadamente 35 ha, situados entre as coordenadas mínimas de $30^{\circ} 06$ ' 25 " $-51^{\circ} 41^{\prime} 15$ " e máximas de $-30^{\circ} 05$ ' 44 " $-51^{\circ} 40$ ' 60 " de latitude sul e longitude oeste, respectivamente. A região estudada apresenta altitude média de 46 m e clima do tipo Cfa, subtropical úmido, conforme classificação climática de Köppen.

O experimento vem sendo conduzido há 22 anos com diferentes intensidades de pastejo, para formação de níveis fixos de oferta de forragem diária. Os tratamentos constam de quatro níveis de ofertas de forragem (kg de matéria seca (MS) /100 kg de peso vivo/dia), expressos em \%: 4, 8, 12 e $16 \%$, as quais são determinadas em função do manejo de diferentes taxas de lotação. Assim, quanto menor a oferta de forragem, maior o número de animais presentes por unidade de forragem disponível. O método de pastejo utilizado é o contínuo com taxa de lotação variável, empregando-se a técnica put and take (Mott \& Lucas, 1952), com ajustes de carga em intervalos de aproximadamente 28 dias a partir de avaliações mensais da quantidade de forragem disponível, conforme procedimentos descritos em Soares et al. (2005). Avaliaramse conjuntamente duas parcelas mantidas em exclusão de pastejo desde o início do experimento, em 1986, e aqui consideradas como testemunha não pastejada. As últimas foram tomadas como referência para fins de cálculos dos índices apresentados. O delineamento utilizado foi de blocos ao acaso, com duas repetições, avaliando-se 10 unidades experimentais.

O solo da área experimental é predominantemente classificado como Argissolo Vermelho distrófico, com distribuição granulométrica de acordo com as proporções: 250, 150, 300, $300 \mathrm{~g} \mathrm{~kg}^{-1}$ de areia grossa, 
areia fina, silte e argila, respectivamente, na profundidade de 0 a $20 \mathrm{~cm}$.

As amostragens de solo foram realizadas em outubro de 2008 e efetuadas em diferentes camadas, em duplicata, para cada parcela. Para avaliação da densidade do solo (DS), coletaram-se amostras indeformadas com anéis de $8,3 \mathrm{~cm}$ de diâmetro e altura de $5 \mathrm{~cm}$, nas camadas de 0 a 5,5 a 10,10 a 15 e 15 a $20 \mathrm{~cm}$, conforme método proposto por Embrapa (1997).

A estabilidade de agregados em água foi determinada nas camadas de 0 a 10 e 10 a $20 \mathrm{~cm}$, separando-se de cada classe a fração areia, utilizandose o método descrito por Kemper \& Chepil (1965). O peneiramento em água distribuiu os agregados nas classes de $>4,76,4,76-2,2-1,1-0,5,0,5-0,25$ e $<0,25 \mathrm{~mm}$. A partir desses valores, obteve-se o diâmetro médio ponderado (DMP), que foi calculado conforme a equação:

$$
\mathrm{DMP}=\Sigma^{\mathrm{n}}{ }_{\mathrm{i}=1}\left(\mathrm{x}_{\mathrm{i}} \cdot \mathrm{w}_{\mathrm{j}}\right)
$$

em que $\mathrm{w}_{\mathrm{i}}=$ proporção (\%) de cada classe em relação ao total e $\mathrm{x}_{\mathrm{i}}=$ diâmetro médio das respectivas classes (mm).

Para determinação da massa de raízes, foram coletadas amostras de solo nas profundidades de $0 \mathrm{a}$ 10 e 10 a $20 \mathrm{~cm}$, com auxílio de um trado calador de $6,5 \mathrm{~cm}$ de diâmetro, nos mesmos locais das demais amostragens. As amostras foram dispersas em água e peneiradas em peneira de $1 \mathrm{~mm}$, para separação das raízes. Depois de separadas, estas foram secas em estufa a $65^{\circ} \mathrm{C}$ e pesadas em balança de precisão com três casas decimais, sendo os resultados expressos em $\mathrm{Mg}$ de raízes secas por hectare.

A amostragem para análise de carbono orgânico total (COT), fração particulada e associada a minerais foi realizada em camadas de $5 \mathrm{~cm}$, até $20 \mathrm{~cm}$ de profundidade. $\mathrm{O}$ fracionamento físico da matéria orgânica foi realizado segundo método de Cambardella \& Elliot (1992). Foram pesados $20 \mathrm{~g}$ de solo e adicionados $80 \mathrm{~mL}$ de solução de hexametafosfato de sódio $\left(5 \mathrm{~g} \mathrm{~L}^{-1}\right)$. As amostras foram agitadas por $18 \mathrm{~h}$ e, posteriormente, passadas em peneira de $53 \mu \mathrm{m}$. O material retido na peneira foi seco em estufa a $50{ }^{\circ} \mathrm{C}$, até atingir massa constante. Posteriormente, foi moído e analisado quanto ao seu teor de C orgânico, denominado de $\mathrm{C}$ orgânico particulado (COP).

A determinação dos teores de $\mathrm{C}$ total e particulado foi realizada em um analisador Shimadzu TOC-V CSH. Os estoques de COT e C da matéria orgânica particulada (CMOP) no solo foram calculados em massas equivalentes de solo, conforme proposto por Ellert \& Bettany (1995). Foi feito o cálculo do Indice de Manejo do Carbono (IMC) e de seus componentes de acordo com Blair et al. (1995), com as adaptações de Dieckow et al. (2005), o qual considera a MOP como representante da fração lábil da MOS e a matéria orgânica associada aos minerais, como não lábil.
A labilidade do C (LC) foi calculada para a profundidade amostrada (0 a $20 \mathrm{~cm}$ ) como sendo a relação entre a fração de C lábil e a fração de $\mathrm{C}$ não lábil, de acordo com a equação:

$$
\text { LC = C lábil / C não lábil }
$$

em que $\mathrm{C}$ lábil = estoque de $\mathrm{C}$ da fração particulada; $\mathrm{e}$ $\mathrm{C}$ não lábil = estoque de $\mathrm{C}$ da fração associada a minerais.

O índice de manejo de $\mathrm{C}$ (IMC) e seus componentes, o índice de estoque de C (IEC) e o índice de labilidade do C (ILC) foram calculados para a camada de 0$20 \mathrm{~cm}$, considerando como referência o solo das parcelas em exclusão de pastejo $(\mathrm{IMC}=100 \%)$ :

$$
\text { IMC }=\text { IEC } \times \text { ILC x } 100
$$

em que IEC $=$ estoque de $\mathrm{C}$ do tratamento / estoque de $\mathrm{C}$ do tratamento-referência; $\mathrm{e}$ ILC $=\mathrm{LC}$ do tratamento/ LC do tratamento-referência.

Os dados foram submetidos à análise de variância a $5 \%$. Foi realizado teste de regressão polinomial, verificando-se a diferença entre médias ou a significância do coeficiente, utilizando-se o software Excel ${ }^{\circledR}$ e SigmaPlot 10.0.

\section{RESULTADOS E DISCUSSÃO}

O aumento na intensidade de pastejo, com redução da oferta de forragem e aumento do número de animais por área, promoveu elevação na densidade do solo nas camadas de 0 a 5 e 5 a $10 \mathrm{~cm}$, com resposta linear positiva dessa variável com a redução das ofertas testadas (Figura 1). Os menores valores na área sem pastejo são pertinentes, considerando-se que animais imprimem pressão sobre o solo ao deslocarem-se em busca de forragem, promovendo rearranjo de partículas e aumento de densidade do solo (Taboada, 2005). Esse comportamento foi observado por Bertol et al. (1998), em avaliação efetuada em 1993, sete anos após o início do experimento, quando também diagnosticaram aumento da Ds nas maiores intensidades de pastejo na profundidade de 10 a $15 \mathrm{~cm}$.

Os dados deste trabalho demonstram o efeito do pisoteio dos animais sobre a Ds nas camadas superficiais. O mesmo foi observado por Salton et al. (2001), os quais relataram aumento da Ds na camada $0-5 \mathrm{~cm}$ em cerca de $60 \%$ da área da parcela experimental com aveia sob pastejo. No entanto, nesse estudo os pesquisadores verificaram que, para profundidades maiores que $10 \mathrm{~cm}$, a Ds não mais se relacionava com a oferta de forragem, indicando que as alterações causadas na relação massa/volume do solo, pela pressão exercida pelo tráfego animal, deixam de existir. Resultados de outros trabalhos (Flores, 2007; Conte et al., 2007) em Latossolo, em sistema de integração lavoura-pecuária sob semeadura direta, 


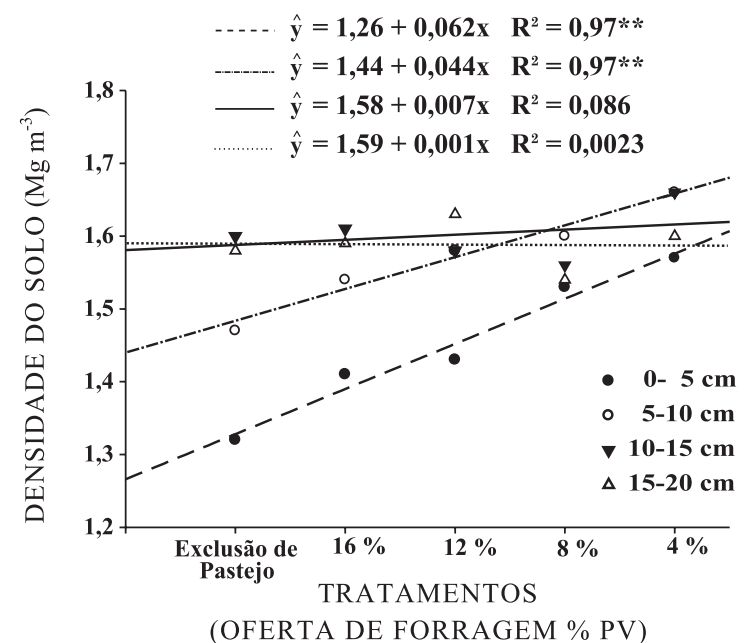

Figura 1. Densidade do solo em função da oferta de forragem em camadas de solo sob pastagem natural por 22 anos.

também demonstram que o efeito de pisoteio animal sobre atributos físicos de solo restringem-se à camada superficial, até o máximo de $12 \mathrm{~cm}$. No presente trabalho (Figura 1), o aumento na intensidade de pastejo apresentou efeito pronunciado sobre a Ds na camada superficial $(0-5 \mathrm{~cm})$, que variou de 1,32 a $1,57 \mathrm{Mg} \mathrm{m}^{-3}$, para a área excluída do pastejo e para a menor oferta (4\%), respectivamente. O intervalo nos valores de Ds, considerando-se as profundidades de amostragem até $20 \mathrm{~cm}$, foi de 1,32 a $1,66 \mathrm{Mg} \mathrm{m}^{-3}$. Esses resultados são similares aos encontrados por Dieckow et al. (2009), que obtiveram Ds de 1,50, 1,57 e $1,63 \mathrm{Mg} \mathrm{m}^{-3}$ para as camadas de $0-5,5-10$ e 10 $20 \mathrm{~cm}$, respectivamente, em um Argissolo sob pastagem nativa, sem controle de oferta de forragem.

Nesta avaliação (Figura 1), a análise de regressão apresentou ajuste $(p<0,05)$ para os dados de ofertas de forragem e Ds nas camadas de 0 a 5 e 5 a $10 \mathrm{~cm}$, diferindo do anteriormente encontrado por Bertol et al. (1998), em que o ajuste foi curvilinear para as camadas de $0-3$ e 3-6 cm, com os menores valores nos níveis intermediários de oferta de forragem (8 e $12 \%$ ) e maiores nos extremos (4 e $16 \%$ ).

A menor Ds nos tratamentos com maior oferta de forragem é reflexo do manejo empregado. A menor pressão aplicada pelos bovinos em pastejo também alterou a estruturação do solo, que apresentou valores mais elevados de DMP de agregados em ambas as camadas (Figura 2), porém somente de forma significativa $(\mathrm{p}<0,05)$ na camada de 0 a $10 \mathrm{~cm}$. Isso, provavelmente, porque o aumento da oferta de forragem promoveu aumento na massa de raízes das espécies vegetais que compunham a pastagem (Figura 3 ) e se refletiu na estruturação do solo. Dessa forma, houve aumento do DMP dos agregados nos tratamentos com maior oferta de forragem e consequente maior pressão de pastejo.
Os valores para DMP encontrados neste estudo, para as duas profundidades de avaliação, são superiores aos verificados na avaliação de 1993 (Bertol et al., 1998) aos sete anos de condução, aplicando o método sugerido por Tisdall et al. (1978). A utilização do DMP como variável isolada, segundo Forsythe (1975), não é um bom indicativo da qualidade estrutural do solo, pois solos compactados podem apresentar elevada estabilidade de agregados em água e, consequentemente, maiores valores de DMP, porém as relações na distribuição entre microporos, macroporos e a porosidade total encontram-se alteradas.

Aumento no DMP de agregados é comumente observado em solos não submetidos a elevadas pressões, conduzidos com manejos que promovam a entrada de biomassa no sistema, promovendo elevação no teor de $\mathrm{C}$ do solo. $\mathrm{O}$ mesmo é válido na presença de

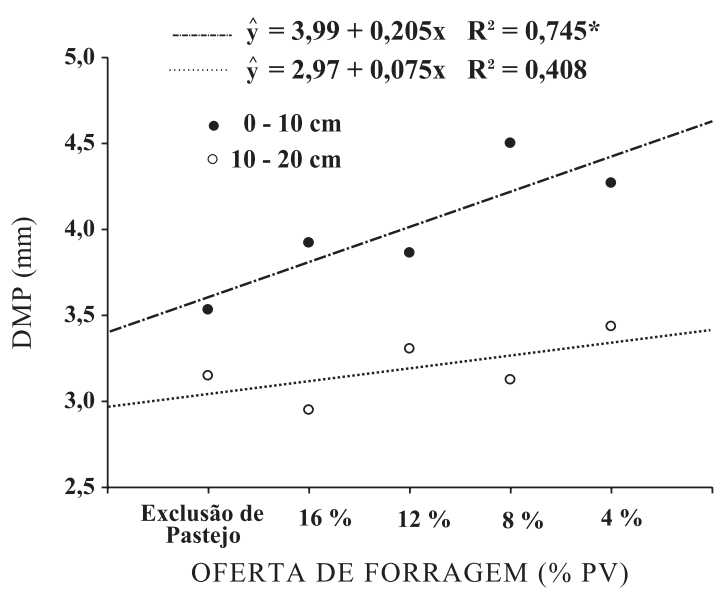

Figura 2. Diâmetro médio ponderado (DMP) dos agregados estáveis em água em camadas de um Argissolo sob pastagem natural submetida a diferentes ofertas de forragem por 22 anos.

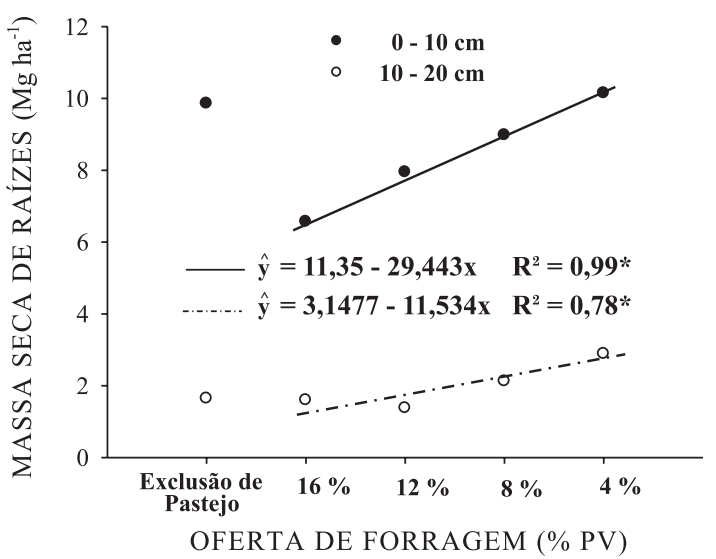

Figura 3. Relação entre a massa seca de raízes em camadas de um Argissolo e a oferta de pastagem natural por 22 anos. 
sistema radicular abundante, principalmente de gramíneas, uma vez que a formação e a estabilidade dos agregados estão ligadas ao crescimento das raízes e à dinâmica da matéria orgânica do solo (Silva \& Mielniczuk, 1997; Salton et al., 2005). O DMP de agregados na área com exclusão de pastejo (Figura 2), aqui considerada como testemunha, foi menor que o encontrado nas áreas pastejadas. Esse efeito é atribuído à agregação do solo promovida, em grande parte, pelo efeito do sistema radicular abundante (Paladini \& Mielniczuk, 1991), sobretudo quando se trata de gramíneas - como é nesse caso - que apresentaram expressiva massa radicular na camada de solo superficial (0 a $10 \mathrm{~cm}$ ) (Figura 3). Na camada superficial, a presença de agregados de maior diâmetro pode estar associada ao maior teor de C orgânico total encontrado, segundo Salton et al. (2008) (Figura 5). Este promove a agregação do solo, conforme demonstrado pelo elevado DMP encontrado, promovido principalmente pela classe de agregados maiores que $4,76 \mathrm{~mm}$, que, para alguns dos tratamentos, ultrapassa $60 \%$ dos agregados estáveis em água. No entanto, nesse caso, acredita-se que prevaleça o efeito de sistema radicular das gramíneas que compõem a pastagem na agregação do solo, sendo mais expressiva com o aumento da massa de raízes conforme se eleva a intensidade de pastejo.

Quanto à massa seca de raízes encontrada (Figura 3), percebe-se menor quantidade na camada de 10 a $20 \mathrm{~cm}$, quando comparada à observada na camada 0 a $10 \mathrm{~cm}$. Isso indica que o sistema radicular das espécies que compõem a pastagem nativa do local, predominantemente gramíneas, concentra-se superficialmente. Dessa forma, elas podem estar promovendo aumento no $\mathrm{C}$ do solo na camada superficial, como demonstrado por Bertol et al. (1998) e Salton et al. (2008), contribuindo, assim, para a agregação do solo, com a elevação do DMP dos agregados. A explicação para a elevada presença de raízes nas camadas superficiais do solo é que a amostragem considera como sendo raízes também os rizomas de Paspalum notatum; com isso, o aumento da massa de raízes pode ser atribuído em parte à crescente participação dessa espécie à medida que se diminui a oferta de forragem (Boldrini, 1993).

Verifica-se (Figura 3) que a quantidade de raízes na área excluída de pastejo supera à encontrada nas menores ofertas de forragem na profundidade $0 \mathrm{a}$ $10 \mathrm{~cm}$. No entanto, quando se consideram os dados de agregação do solo, não se encontra relação dessa quantidade de raízes com os dados de DMP de agregados. A explicação para isso deve estar no tipo de sistema radicular encontrado nesse tratamento. Como a testemunha foi excluída do pastejo por 22 anos, ocorreu uma sucessão diferenciada de espécies, e atualmente predomina uma vegetação arbustiva com pequenas árvores, de diversas espécies e tamanho, podendo estas atingir de 5 a $8 \mathrm{~m}$ de altura. Com isso, o sistema radicular presente é totalmente diverso do encontrado nos tratamentos de oferta de forragem em campo nativo. Na testemunha a quantidade de raízes superficiais é menor, e o diâmetro destas, maior, a ponto de no momento da separação terem sido encontrados segmentos de raízes com diâmetro de aproximadamente $5 \mathrm{~mm}$. Dessa forma, é possível entender a elevada massa de raízes determinada. Certamente, se estivesse sendo avaliado o número de raízes ou o seu comprimento, a correlação com agregação do solo seria significativa e explicaria o baixo DMP de agregados encontrado. Assim, justificase a aplicação de regressão somente envolvendo as quatro ofertas de forragem em questão $(4,8,12$ e $16 \% \mathrm{PV})$.

O estoque e as respectivas frações de C orgânico do solo não foram alterados pelos tratamentos de oferta de forragem (Figura 4) quando submetidos à análise de variância. Percebe-se, no entanto, que em todos os tratamentos há predomínio da fração ligada aos minerais (CAM). Sabe-se porém que, mesmo em menor quantidade, a fração particulada (CMOP) é muito dinâmica no solo e exerce importante papel de agregação e nos ciclos biológicos no solo. Essa também é a fração mais sensível aos manejos impostos ao solo ou ao sistema produtivo (Tomazi, 2008). A distribuição do COT em profundidade corrobora com a encontrada por Dieckow et al. (2009) para condição de pastagem nativa em Argissolo na mesma região.

A quantidade de COT decresce na camada avaliada na ordem decrescente de oferta de forragem: testemunha, 12, 8, 4 e $16 \%$. Destaca-se o tratamento com oferta de $16 \%$, em que a quantidade de biomassa de forragem é maior e o COT medido é também menor neste experimento, em todas as profundidades analisadas, podendo ser associado à quantidade de raízes encontradas.

No quadro 1 são apresentados os dados de labilidade do $\mathrm{C}$, índice de labilidade do $\mathrm{C}$, índices de estoque de $\mathrm{C}$ e índice de manejo de C. O IMC, por ser o produto entre o IEC e o ILC, reflete a qualidade dos sistemas

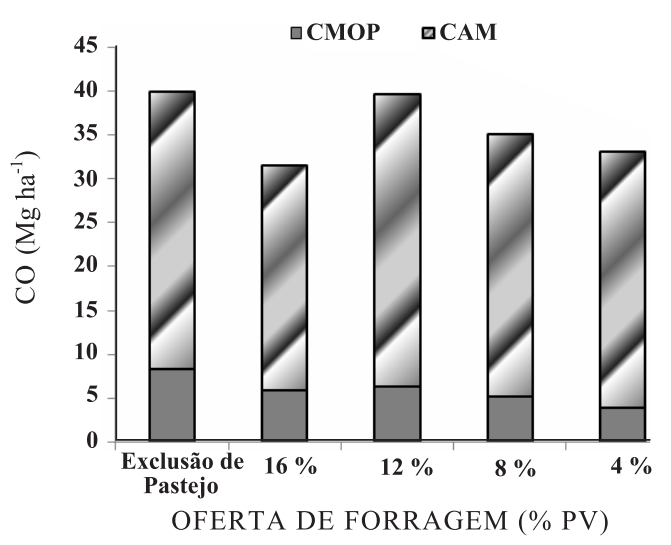

Figura 4. Carbono da matéria orgânica particulada (CMOP) e carbono associado a minerais (CAM) em função da oferta de forragem em pastagem nativa por 22 anos. 


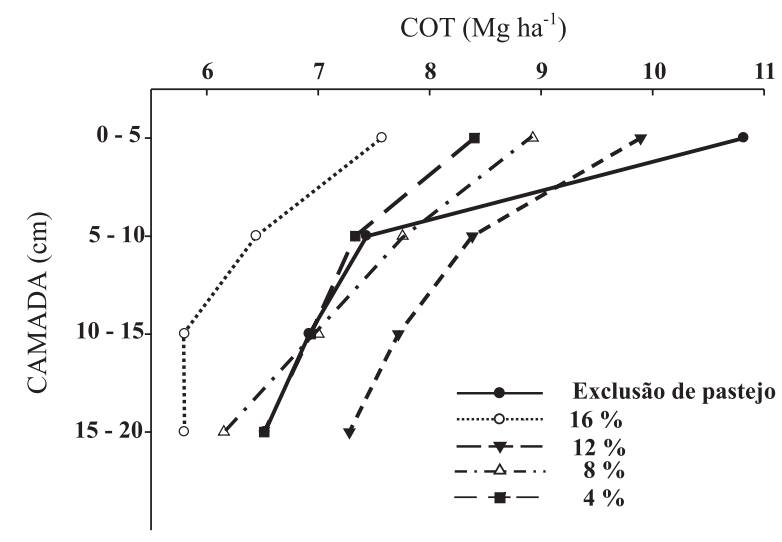

Figura 5. Carbono orgânico total em função da profundidade de amostragem e da oferta de forragem em pastagem nativa por 22 anos.

de manejo, permitindo inferir a respeito da sustentabilidade do sistema em questão (Blair et al., 1995; Dieckow et al., 2005; Souza et al., 2009). O IEC expressa a razão entre o estoque de $\mathrm{C}$ do solo em cada tratamento de oferta de forragem e o solo da área testemunha, e o ILC, a razão entre a labilidade do C do solo em cada tratamento de oferta de forragem e o solo-referência, ambos para a camada de 0-20 cm.

O aumento no C lábil é um fator relevante para a qualidade do solo, pois, segundo Christensen (1992), esta é a fração mais dinâmica do $\mathrm{C}$ do solo, sendo usada como fonte de energia para o sistema solo-planta, retroalimentando os processos químicos, físicos e biológicos e favorecendo as interações entre os componentes do sistema solo, contribuindo assim para a expressão das propriedades emergentes do sistema (Tomazi, 2008). Dessa forma, também promove a agregação e a estruturação do solo (Salton et al., 2008), comprovada pelos elevados valores de DMP (Figura 2). Neste experimento, a LC decresce com a redução na oferta de forragem, assim como o ILC. No entanto, o IEC mostra que o tratamento com $12 \%$ de oferta de forragem acumulou mais $\mathrm{C}$ que a testemunha (área de exclusão de pastejo), seguido pelos tratamentos 8 e
$4 \%$, com o tratamento $16 \%$ demonstrando a menor capacidade de acúmulo de $\mathrm{C}$.

Com exceção da oferta de forragem de $12 \%$, temse menor potencial de acúmulo de $\mathrm{C}$ nos demais tratamentos em relação à área testemunha. Já o IMC, usado para expressar a qualidade do sistema de manejo, indica que os tratamentos de 16 e $12 \%$ de oferta de forragem são similares quanto a esse índice, mantendo-se mais próximo da testemunha. Nos demais tratamentos, o IMC decresce de acordo com a oferta de forragem, atingindo um valor muito baixo $(39,7 \%)$ no tratamento com menor oferta de forragem, indicando substancial perda de qualidade do solo nesse manejo, com riscos para a sustentabilidade do sistema produtivo, conforme apontado por Dieckow et al. (2005), Dieckow et al. (2009) e Souza et al. (2009), usando o IMC como indicador.

\section{CONCLUSÕES}

1. A utilização das pastagens naturais com bovinos promove alterações nos atributos físicos e da agregação na camada superficial do solo, demonstrando relação com a oferta de forragem.

2. A massa de raízes da pastagem nativa e o diâmetro médio de agregados do solo elevam-se de forma linear com o aumento na pressão de pastejo.

3. $\mathrm{O}$ índice de manejo de $\mathrm{C}$ demonstra que as maiores ofertas de forragem (12 e $16 \%$ ) proporcionam melhor qualidade ao sistema em pastagem natural, especialmente em relação à menor oferta (4\%), com baixa sustentabilidade.

\section{LITERATURA CITADA}

BAYER, C.; MARTIN-NETO, L.; MIELNICZUK, J. \& PAVINATO, A. Armazenamento de carbono em frações lábeis da matéria orgânica de um Latossolo Vermelho sob plantio direto. Pesq. Agropec. Bras., 39:677-683, 2004.

Quadro 1. Labilidade da matéria orgânica (LC), índice de labilidade (ILC), índice de estoque de carbono (IEC) e índice de manejo de carbono (IMC) em Argissolo sob pastagem nativa com diferentes níveis de oferta de forragem por 22 anos

\begin{tabular}{lccccc}
\hline \multirow{5}{*}{ Índice } & \multicolumn{2}{c}{ Ofertas de Forragem (\% PV) } \\
\cline { 2 - 5 } & Exclusão de pastejo & $\mathbf{1 6} \%$ & $\mathbf{1 2} \%$ & $\mathbf{8} \%$ & $\mathbf{4} \%$ \\
\hline LC & $0,35 \mathrm{a}$ & $0,30 \mathrm{~b}$ & $0,23 \mathrm{c}$ & $0,21 \mathrm{c}$ & $0,15 \mathrm{~d}$ \\
ILC & 1,00 & $0,86 \mathrm{a}$ & $0,66 \mathrm{~b}$ & $0,60 \mathrm{~b}$ & $0,43 \mathrm{c}$ \\
IEC & 1,00 & $0,81 \mathrm{c}$ & $1,05 \mathrm{a}$ & $0,94 \mathrm{~b}$ & $0,92 \mathrm{~b}$ \\
IMC & 100 & $69,2 \mathrm{a}$ & $69,5 \mathrm{a}$ & $56,2 \mathrm{~b}$ & $39,7 \mathrm{c}$ \\
\hline
\end{tabular}

Letras na horizontal indicam diferença pelo teste de Tukey a $5 \%$. 
BERTOL, I.; GOMES, K.E.; DENARDIN, R.B.N.; MACHADO, L.A.Z. \& MARASCHIN, G.E. Propriedades físicas do solo relacionadas a diferentes níveis de oferta de forragem numa pastagem natural. Pesq. Agropec. Bras., 33:779786,1998

BLAIR, G.J.; LEFROY, R.D.B. \& LISLE, L. Soil carbon fractions based on their degree of oxidation, and the development of a carbon management index, for agricultural systems. Austr. J. Agric. Res., 46:1459-1466, 1995.

BODDEY, R.M.; JANTALIA, C.P.; CONCEICÃO, P.C.; ZANATTA, J.A.; BAYER, C.; MIELNICZUK, J.; DIECKOW, J.; SANTOS, H.P. DENARDIN, J.E.; AITA, C.; GIACOMINI, S.J.; ALVES, B.J.R. \& URQUIAGA, S. Carbon accumulation at depth in Ferralsols under zerotill subtropical agriculture. Glob. Chan. Biol., 16:784-795, 2010.

BOLDRINI, I.I. Dinâmica da vegetação de uma pastagem natural sob diferentes níveis de oferta de forragem e tipos de solos, Depressão Central, RS. Porto Alegre, Universidade Federal do Rio Grande do Sul, 1993. 262p. (Tese de Doutorado)

BOLDRINI, I.I. Campos do Rio Grande do Sul: caracterização fisionômica e problemática ocupacional. Porto Alegre, Universidade Federal do Rio Grande do Sul, 1997. 39p. (Instituto de Biociências. Boletim, 56).

CAMBARDELLA, C.A. \& ELLIOT, E.T. Particulate soil organic matter changes across a grassland cultivation sequence. Soil Sci. Soc. Am. J., 56:777-783, 1992.

CARVALHO, P.C.F. Access to land, livestock production and ecosystem conservation in the Brazilian Campos biome: Tthe natural grasslands dilemma. Disponível em: http:// $\mathrm{w}$ w w. i c a r d . o r g/e n/e ve n to s/t e m/ STS\%20UFGRS\%20Biome.pdf. acesso em: 27 dez. 2009.

CHRISTENSEN, B.T. Physical fractionation of soil and organic matter in primary particle size and density separates. Adv. Soil Sci., 20:1-90, 1992.

CONTE, O.; LEVIEN, R.; TREIN, C.R.; CEPIK, C.T.C. \& DEBIASI, H. Demanda de tração em haste sulcadora na integração lavoura-pecuária com diferentes pressões de pastejo e sua relação com o estado de compactação do solo. Eng. Agric., 27:220-228, 2007.

DIECKOW, J.; BAYER, C.; CONCEIÇÃO, P.C.; ZANATTA, J.A.; MARTIN-NETO, L.; MILORI, D.M.B.P.; SALTON, J.C.; MACEDO, M.C.M.; MIELNICZUK, J. \& HERNANI, L.C. Land use, tillage, texture and organic matter stock and composition in tropical and subtropical Brazilian soils. Europ. J. Soil Sci., 60:240-249, 2009.

DIEKOW, J.; MIELNICZUK, J.; KNICKER, H.; BAYER, C.; DICK, D.P. \& KNABNER, I.K. Carbon and nitrogen stocks in physical fractions of a subtropical Acrisol as influenced by long-term no-till cropping systems and $\mathrm{N}$ fertilization. Plant Soil, 268:319-328, 2005.

ELLERT, B.H. \& BETTANY, J.R. Calculation of organic matter and nutrients stored in soils under contrasting management regimes. Canadian J. Soil Sci., 75:529-538, 1995.
EMPRESA BRASILEIRA DE PESQUISA AGROPECUÁRIA EMBRAPA. Manual de métodos de análises de solo. 2.ed. Rio de Janeiro, Ministério da Agricultura e do Abastecimento, 1997. 212p.

FLORES, J.P.C.; ANGHINONI, I.; CASSOL, L.C.; CARVALHO, P.C.F.; LEITE, J.G.D. \& FRAGA, T.I. Atributos físicos do solo e rendimento de soja em sistema plantio direto em integração lavoura-pecuária com diferentes pressões de pastejo. R. Bras. Ci. Solo, 31:771-780, 2007.

FORSYTHE, W. Fisica de suelos; manual de laboratório. New York, University Press, 1975. 324p.

GONÇALVES, E.N. Comportamento ingestivo de bovinos e ovinos em pastagem natural da Depressão Central do Rio Grande do Sul. Porto Alegre, Universidade Federal do Rio Grande do Sul, 2007. 138p. (Tese de Doutorado)

KEMPER, W.D. \& CHEPIL, W.S. Size distribution of aggregates. In: BLACK, C.A., ed. Methods of soil analysis: Physical and mineralogical properties, including statistics of measurement and sampling. Madison, American Society of Agronomy, 1965. v.1. p.499-510.

MARSCHNER, H. Mineral nutrition of higher plants. 2.ed. London, Academic Press, 1995. p.508-536.

MOTT, G.O. \& LUCAS, H.L. The desing, conduct, and interpretation of grazing trials on cultivated and improved pastures. In: INTERNATIONAL GRASSLAND CONGRESS, 6., Pasadena, 1952. 'Proceedings... Pasadena, 1952.

MULLER, M.M.L.; GUIMARÃES, M.F.; DESJARDINS, T. \& MARTINS, P.F.S. Degradação de pastagens na Região Amazônica: Propriedades físicas do solo e crescimento de raízes. Pesq. Agropec. Bras., 36:1409-1418, 2001.

PALADINI, F.L.S. \& MIELNICZUK, J. Distribuição de tamanho de agregados de um solo Podzólico Vermelho-Escuro afetado por sistemas de culturas. R. Bras. Ci. Solo, 15:135140,1991

PULLEMAN, M.M.; SIX, J.; van BREEMEN, N. \& JONGMANS, A.G. Soil organic matter distribution and microaggregate characteristics as affected by agricultural management and earthworm activity. Europ. J. Soil Sci., 56:453-467, 2005 .

ROSOLEM, C.A.; VALE, L.S.R.; GRASSI FILHO, H. \& MORAES, M.H. Sistema radicular e nutrição do milho em função da calagem e da compactação do solo. R. Bras. Ci. Solo, 18:491-497, 1994.

SALTON, J.C.; MELLO, N.A.; MATSUOKA, M.; CARVALHO, P.C.F.; NABINGER, C.; BAYER, C. \& MIELNICZUK, J. Atributos físicos de um Argissolo sob pastagem natural após 18 anos sob diferentes níveis de ofertas de forragem. R. Ci. Agrovet., 7:107-118, 2008.

SALTON, J.C.; FABRÍCIO, A.C.; MACHADO, L.A.Z. \& OLIVEIRA, H. Pastoreio da aveia e compactação do solo. Dourados, Embrapa Agropecuária Oeste, 2001. 5p. (Embrapa Agropecuária Oeste. Comunicado Técnico, 48). 
SALTON, J.C.; MIELNICZUK, J.; BAYER, C.; FABRICIO, A.C.; MACEDO, M.C.M.; BROCH, D.L.; BOENI, M. \& CONCEIÇÃO, P.C. Matéria orgânica do solo na integração lavoura-pecuária em Mato Grosso do Sul. Dourados, Embrapa Agropecuária Oeste, 2005. 58p. (Boletim de Pesquisa e Desenvolvimento, 29).

SILVA, I.F. \& MIELNICZUK, J. Ação do sistema radicular de planta na formação e estabilização de agregados do solo. R. Bras. Ci. Solo, 21:113-117, 1997.

SOARES, A.B.; CARVALHO, P.C.F.; NABINGER, C.; SEMMELMANN, C.; TRINDADE, J.K.; GUERRA, E.; FREITAS, T.S.; PINTO, C.E.; FONTOURA JÚNIOR, J.A \& FRIZZO, A. Produção animal e de forragem em pastagem nativa submetida a distintas ofertas de forragem. Ci. Rural, 35:1148-1154, 2005.

SOUZA, E.D.; COSTA, S.E.V.G.A.; ANGHINONI, I.; CARVALHO, P.C.F.; ANDRIGUETI, M. \& CAO, E. Estoques de carbono orgânico e de nitrogênio no solo em sistema de integração lavourapecuária em plantio direto, submetido a intensidades de pastejo. R. Bras. Ci. Solo, 33:1829-1836, 2009.
TABOADA, M.A. Efectos del pastoreo y pisoteo animal sobre lãs propriedades física de suelos. In: JORAJURÍA COLLAZO, D., org. Reologia del suelo agrícola bajo tráfico: Modificaciones fisico-mecánicas del suelo vinculadas a la compactación debida al tráfico agrario. La Plata, Universidad Nacional de La Plata, 2005. p.131-43.

TISDALL, J.M.; COCKROFT, B. \& UREN, N.C. The stability of soil aggregates as affected by organic materials, microbial activity and physical disruption. Austr. J. Soil Res., 16:9-17, 1978.

TOMAZI, M. Estabilidade da matéria orgânica em Latossolos do Cerrado sob sistemas de uso e manejo. Porto Alegre, Universidade Federal do Rio Grande do Sul, 2008. 108p. (Tese de Doutorado)

TREIN, C.R.; COGO, N.P. \& LEVIEN, R. Métodos de preparo do solo na cultura do milho e ressemeadura do trevo na rotação aveia+trevo/milho, após pastejo intensivo. R. Bras. Ci. Solo, 15:105-111, 1991. 
